М. Эргашов ${ }^{1}$, Х. А. Бабаханова ${ }^{1}$, У. Т. Абдуллаев ${ }^{1}$, А. А. Садриддинов ${ }^{1}$, И. Г. Громыко

${ }^{1}$ Ташкентский институт текстильной и легкой промышленности

${ }^{2}$ Белорусский государственный технологический университет

\title{
МЕТОД ОПРЕДЕЛЕНИЯ \\ ДЕФОРМАЦИОННЫХ И ПРОЧНОСТНЫХ СВОЙСТВ БУМАГИ
}

В статье предлагаются методы теоретического и экспериментального исследования для оценки прочности бумаги на изгиб. Приведены алгоритм расчета и результаты числовых экспериментальных исследований зависимости деформации изгиба от основных деформационных показателей бумаги.

Проведенные исследования позволили получить данные, по которым были определены форма кривой и численные значения прогиба образцов бумаги. Также было установлено, что вектор силы собственного веса всегда направлен вертикально вниз. В случае, если бумага имеет однородную структуру и объемную плотность, то сила собственного веса будет равномерно распределена по всему объему образца. Учитывая различный состав бумаги, можно отметить, что момент начала изгиба и кривые изгиба будут различаться. Установленные из эксперимента значения времени начала изгиба, геометрическая форма и координаты поверхности изгиба могут быть использованы для оценки свойств бумаги и бумажных изделий. Предложенный метод исследования деформационных и прочностных свойств бумаги позволит прогнозировать ее свойства на стадии изготовления, а также учитывать возможность ее использования в конкретных условиях печати, что обеспечит получение полиграфической продукции высокого качества.

Ключевые слова: бумага, прочность на изгиб, деформация, коэффициент жесткости на изгиб, объемная плотность.

Для цитирования: Эргашов М., Бабаханова Х. А., Абдуллаев У. Т., Садриддинов А. А., Громыко И. Г. Метод определения деформационных и прочностных свойств бумаги // Труды БГТУ. Сер. 4, Принт- и медиатехнологии. 2021. № 2 (249). С. 11-17.

\section{Ergashov ${ }^{1}$, Kh. A. Babakhanova ${ }^{1}$ U. T. Abdullayev ${ }^{1}$, A. A. Sadriddinov ${ }^{1}$, I. G. Gromyko ${ }^{2}$ ${ }^{1}$ Tashkent Institute of Textile and Light Industry ${ }^{2}$ Belarusian State Technological University \\ METHOD FOR DETERMINING THE DEFORMATION AND THE STRENGTH PROPERTIES OF THE PAPER}

The article offers methods of theoretical and experimental research to assess the bending strength of paper. The calculation algorithm and the results of numerical experimental studies of the dependence of the bending deformation on the main deformation parameters of the paper are presented. The conducted studies allowed us to obtain data on which the shape of the curve and the numerical values of the deflection of paper samples were determined. It was also established that the vector of the force of its own weight is always directed vertically downwards. If the paper has a uniform structure and volume density, then the force of its own weight will be evenly distributed over the entire volume of the sample. Given the different composition of the paper, it can be noted that the moment of the beginning of the bend and the bending curves will differ. The values of the bending start time, the geometric shape and the coordinates of the bending surface established from the experiment can be used to evaluate the properties of paper and paper products. The proposed method for studying the deformation and strength properties of paper will make it possible to predict its properties at the manufacturing stage, as well as to take into account the possibility of its use in specific printing conditions, which will ensure the production of high quality printing.

Key words: paper, bending strength, deformation, bending stiffness coefficient, bulk density.

For citation: Ergashov M., Babakhanova Kh. A., Abdullayev U. T., Sadriddinov A. A., Gromyko I. G. Method for determining the deformation and the strength properties of the paper. Proceeding of BSTU, issue 4, Print- and Mediatechnologies, 2021, no. 2 (249), pp. 11-17 (In Russian).

Введение. К капиллярно-пористой бумаге, являющейся одним из основных материалов для печати, предъявляются определенные требования и нормы, отклонения от которых свыше допустимого предела приводят к дефектам при воспроизведении. Например, неравномерность объемной плотности по ширине и длине бумажного материала вызывает неравномерность 
влажности, прочности, плотности. Это способствует разрыву бумажного полотна из-за низкой плотности бумаги, а также приводит к избыточному расходу краски при печати. Слишком высокая плотность бумаги вызывает перерасход растительных волокон и делает материал более тяжелым.

Бумага в процессе печатания под воздействием внешних климатических условий и механических нагрузок испытывает деформацию растяжения, сжатия, изгиба и т. д. Сопротивление деформированию определяется сопротивлением сдвига растительных волокон, являющихся основными компонентами бумажной массы, приобретающих новые физические свойства в результате влияния технологических стадий изготовления бумаги, такие как роспуск, размол, сушка, отделка и т. д. [1]. Используемые для оценки величины сопротивления временные напряжения исчезают после снятия внешней нагрузки [2]. Остаточные напряжения, возникающие и уравновешивающиеся в пределах тела без действия внешней нагрузки, сохранятся в волокнистых материалах и будут иметь различные значения и направления действия. В совокупности остаточные напряжения каждого из компонентов бумажной массы характеризуют степень распределения и направленность остаточных напряжений в элементарных объемах материала бумаги.

Неравномерность объемной плотности и остаточные напряжения в материале способствуют появлению таких негативных явлений, как складки, морщины, пузыри, дырки, матовые полосы, соринки, неоднородная толщина, косина листов, а также неравномерному распределению прочностных показателей материала в целом [3].

В промышленных условиях во многих случаях под воздействием химических реагентов (например, различных красящих веществ), влажности, температуры и механических нагрузок степень неравномерности распределения объемной плотности и остаточных напряжений может возрастать [4-7].

До настоящего времени ведутся работы, целью которых является выявление влияния неравномерного распределения объемной плотности на деформационные свойства целлюлознобумажных материалов при растяжении, сжатии или изгибе.

Основная часть. В данной работе предлагается новая методика теоретико-экспериментальной оценки влияния неравномерного распределения объемной плотности на прочностные свойства бумаги.

Общий вид экспериментальной установки стенда для исследования жесткости на изгиб бумажных материалов представлен на рис. 1.

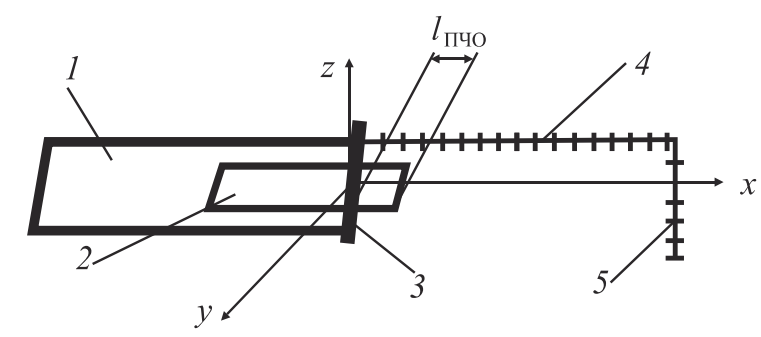

Рис. 1. Схема установки для измерения прогиба бумажного материала

Стенд включает столик 1 с горизонтально расположенной и гладкой поверхностью, на который помещается образец бумаги 2. Вращающийся барабан 3 предназначен для прижима и одновременно передвижения образца бумаги по поверхности столика. Для измерения длины свободной от поверхности стола части образца

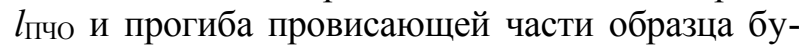
маги используются линейки 4 и 5 с метками.

Барабан на установке выполняет следующие основные функции:

- прижимает образец бумаги к правой границе столика так, чтобы прогиб $z(x)$ и угол $\theta(x)$ поперечных сечений образца бумаги на границе столика равнялись нулю. Эти условия используются в дальнейшем при определении распределения прогиба вдоль горизонтальной оси $x[5]$;

- при вращении вокруг собственной оси поперечного сечения в направлении против часовой стрелки обеспечивает перемещение образца бумаги по поверхности столика;

- вращение с заданной скоростью обеспечивает движение свободной от поверхности столика части бумаги в различных режимах (статическом и динамическом) и производит остановку движения в заданные моменты времени.

Движение барабана с заданной скоростью позволяет устанавливать зависимость прогиба от скорости возрастания собственного веса свободной части бумаги.

Показатели времени остановки движения применяются в целях сравнения законов изменения кривых для различных бумажных материалов.

Следующий этап исследования был посвящен анализу влияния свойств и плотности материалов на прочность бумаги. В некоторый момент времени движения в свободной от поверхности стола части бумаги длиной $l_{\text {пчо }}$ начинает появляться прогиб относительно плоскости $(x, z)$.

Форма кривой и величина прогиба в каждой точке $x$ имеют свои собственные значения, обе эти величины являются функциями координаты $x$. Кроме того, величина прогиба $z(x)$ в каждой точке $x$ обратно пропорциональна 
значению коэффициента жесткости $E J_{Y}$ материала:

$$
z(x) \sim \frac{1}{E J_{Y}},
$$

где $E$ - модуль эластичности (модуль Юнга) материала бумаги, $\mathrm{H} / \mathrm{m}^{2} ; J_{Y}$ - осевой момент инерции $\left(\mathrm{M}^{4}\right)$ поперечного сечения образца относительно оси $y$ (рис. 2).

$$
J_{Y}=\frac{b h^{2}}{12},
$$

где $b$ - длина образца, мм; $h$ - толщина, мм.

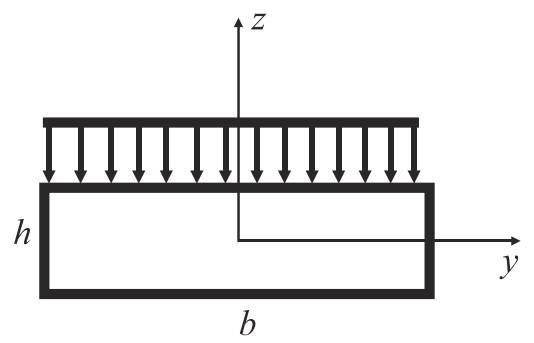

Рис. 2. Схема действия силы собственного веса в поперечном сечении образца бумаги

Зависимость прогиба бумаги от коэффициента жесткости материала представлена на рис. 3.

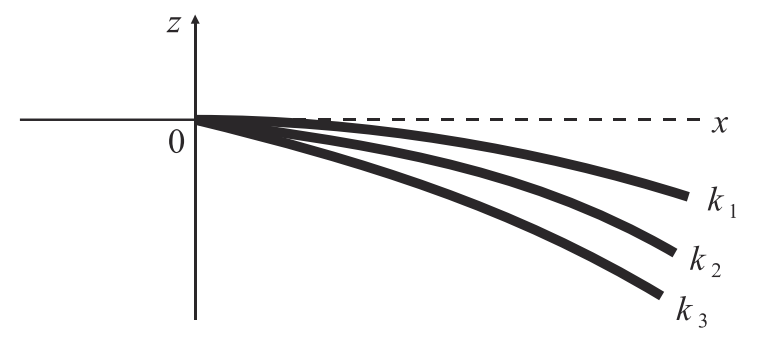

Рис. 3. Зависимость прогиба бумаги от коэффициента жесткости материала

Форма кривой и значения прогиба в каждой точке в свободной от стола части образца бумаги зависят от коэффициента жесткости материала - возрастание величины коэффициента жесткости $E J_{Y}$ приводит к уменьшению значения прогиба. Рассмотрим три образца бумаги, изготовленных из различных материалов, имеющих одинаковые объемные плотности и обладающих различными коэффициентами жесткости на изгиб. Пронумеруем рассматриваемые образцы бумаги условными порядковыми номерами 1, 2, 3 и обозначим их коэффициенты жесткости на изгиб через $k_{i}=\left(E J_{Y}\right)_{i}$, где $i=1,2,3$.

Пусть для коэффициентов $k_{i}$ выполняется следующее условие $k_{1}>k_{2}>k_{3}$. Тогда графики прогиба материалов с порядковыми номерами 1 , 2 и 3 качественно совпадают с кривыми, изображенными на рис. 3. Наибольшие значения прогиба соответствуют материалу бумаги с порядковым номером 3 , т. е. бумаге с наименьшим значением коэффициента жесткости на изгиб материала. Это согласуется с известным в литературе утверждением о том, что возрастание коэффициента жесткости приводит к убыванию прогиба материала [4].

В зависимости от плотности, жесткости на изгиб и других показателей время $t_{\text {нп }}$, соответствующее началу прогиба в свободной части различных видов бумаги, будет отличаться. Очевидно, что возрастание жесткости и плотности материала приводит к возрастанию времени, соответствующему началу прогиба, а убывание этих величин, наоборот, - убыванию времени начала прогиба. Поэтому время $t_{\text {нп, }}$ соответствующее началу прогиба, может служить одним из основных показателей, характеризующих жесткость на изгиб и прочность материала бумаги в целом. Кривая прогиба бумаги представлена на рис. 4.

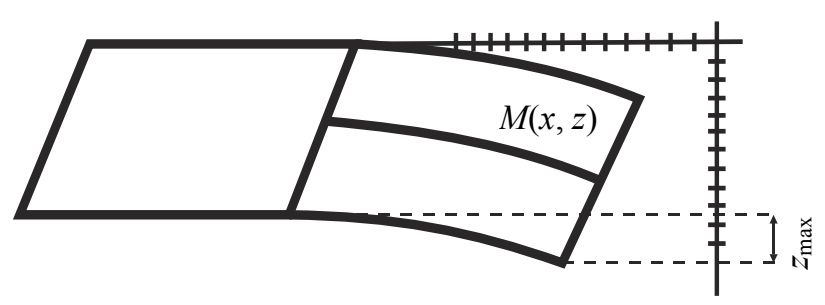

Рис. 4. Кривая прогиба бумаги

Как было отмечено выше, форма и величина поверхности прогиба в каждой точке $M(x, z)$ пространства $(x, y, z)$ имеют соответствующие свойствам каждой бумаги значения (рис. 3). Используя экспериментальные значения координаты точек поверхности прогиба, можно устанавливать прочностные характеристики материала бумаги.

Установленные из эксперимента значения времени $t_{\text {нп }}$ соответствующие началу прогиба, геометрическая форма и координаты поверхности прогиба могут быть применены для оценки свойства материалов бумаги и бумажных изделий.

Исследование внутренних сил, возникающих в поперечных сечениях образца бумаги, показало, что прогиб образца происходит под действием силы собственного веса. Вектор распределенного по всему объему силы собственного веса всегда направлен вертикально вниз, и если бумага имеет однородную равномерную структуру и объемную плотность, то сила собственного веса также будет равномерно распределена по всему объему образца (рис. 2). Интенсивность или объемный вес, приходящий на единицу объема $b h d x$ распределенной силы собственного веса, характеризуется параметром $\gamma$, величина которого определяется экспериментально путем взвешивания элементарного объема 
бумаги. Данный параметр, например, для металла имеет значение $\gamma=7,8 \cdot 10^{-3} \mathrm{\kappa г} / \mathrm{cm}^{3}$, или $\gamma=8,8 \cdot 10^{-3} \mathrm{H} / \mathrm{M}^{3}$.

Суммарная величина силы собственного веса, перерезывающей силы в данном случае, определяется интегрированием величины $G(x)=\int \gamma b h d x$. Интегрируя в пределах от 0 до $l_{\text {пчо, получаем }}$

$$
G_{\max }=\gamma b h l_{\Pi ч О} .
$$

Таким образом, если бумага имеет постоянную объемную плотность и массу, приходящуюся на единицу объема, то перерезывающая сила вдоль ширины образца распределена равномерно, а вдоль длины - по линейно убывающему закону - убывает от наибольшей величины в точке $x=0$ до нуля в точке $x=l_{\text {пчо }}$ (рис. 5).

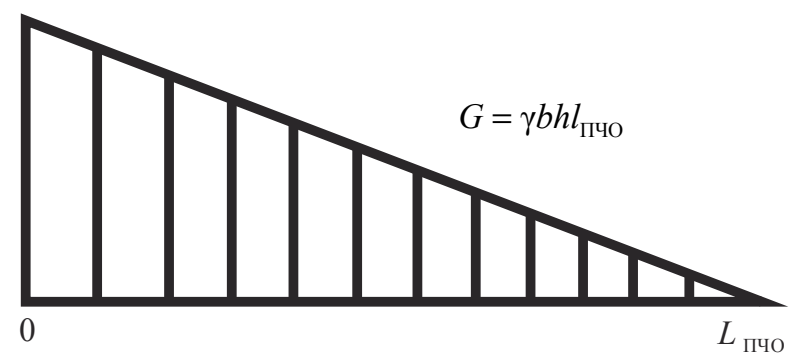

Рис. 5. Эпюра перерезывающей силы

Под действием силы собственного веса в поперечных сечениях образца возникает изгибающий момент $M_{x y}$. При принятых выше допущениях изгибающий момент (плотность и масса вдоль ширины образца распределены равномерно) вдоль ширины образца имеет постоянное значение $M_{x}=m_{0 x} b$, где $m_{0 x}-$ величина изгибающего момента, приходящегося на единицу длины $b$.

В данном случае значения изгибающего момента вдоль длины участка $0 \leq x \leq l_{\text {пчо меня- }}$ ются по параболическому закону:

$$
M_{x}(x)=m_{x} \frac{\left(l_{\Pi ч \mathrm{O}}-x\right)^{2}}{2} .
$$

В сечении $x=0$ изгибающий момент принимает следующее значение:

$$
M_{x}(0)=m_{x} \frac{\left(l_{\Pi}\right)^{2}}{2} .
$$
вен 0 .

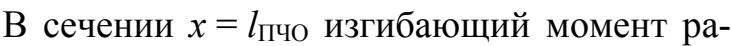

Эпюра изгибающего момента представлена на рис. 6.

Таким образом, под действием силы собственного веса в поперечных сечениях бумаги возникают перерезывающая сила $Q_{x}$ и изгибающий момент $M_{x}$.

На следующем этапе исследования определяли деформацию изгиба образца. В механике при статической нагрузке для расчета значения деформации $z(x)$ и угла поворота $\theta(x)=d z(x) / d x$, возникающих в поперечных сечениях балки, используют метод универсальных уравнений [5].

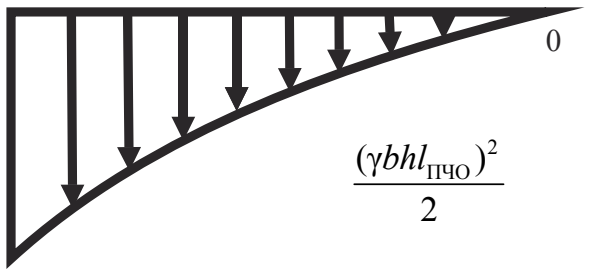

Рис. 6. Эпюра изгибающего момента

В данном случае барабан левой границы об-

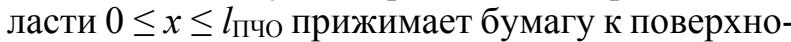
сти столика. Поэтому можно допустить, что в сечении $x=0$ имеют место следующие условия: $\theta(0)=0$ и $z(0)=0$. Отсюда получаем следующие универсальные уравнения:

$$
\begin{aligned}
& z(x)=\frac{1}{E J_{Y}}\left(m_{x} \frac{\left(l_{\Pi Ч О}\right)^{2}}{2} \frac{x^{2}}{2}+\right. \\
& \left.+\gamma b h l_{\Pi ч О} \frac{x^{3}}{6}-\gamma b h \frac{x^{4}}{24}\right) ; \\
& \Theta(x)=\frac{1}{E J_{Y}}\left(m_{x} \frac{\left(l_{\Pi ч О}\right)^{2}}{2} x+\right. \\
& \left.+\gamma b h l_{\Pi Ч О} \frac{x^{2}}{2}-\gamma b h \frac{x^{3}}{6}\right) .
\end{aligned}
$$

Для удобства дальнейшего анализа и проведения числовых экспериментальных исследований равенства (6) и (7) представим в виде

$$
\begin{aligned}
& z(x)=\xi\left(\frac{\left(l_{\Pi ч О} x\right)^{2}}{4}+l_{\text {ПчО }} \frac{x^{3}}{6}-\frac{x^{4}}{24}\right) ; \\
& \Theta(x)=\xi\left(\frac{\left(l_{\Pi ч О}\right)^{2}}{2} x+l_{\text {ПчО }} \frac{x^{2}}{2}-\frac{x^{3}}{6}\right),
\end{aligned}
$$

где

$$
\xi=\frac{\gamma b h}{E J_{Y}} .
$$

Если известны значения параметров $E$ и $J_{Y}$, то, задавая численные значения переменной $x$ из области $0 \leq x \leq l_{\Pi ч о}$, можно построить графики изменения функций $z(x)$ и $\theta(x)$.

Из выражений (6) и (7) следует, что увеличение объемной массы, плотности или площади поперечного сечения (толщины) бумаги способствует увеличению деформации изгиба $z(x)$ и $\theta(x)$. Отсюда вытекает, что кривые $1-3$ на рис. 3 соответствуют случаю $k_{3}>k_{2}>k_{1}$.

Таким образом, виды бумаги, изготовленные из различных материалов, при равных условиях 
провисания изгибаются по различным законам время, соответствующее моменту начала изгиба, и кривые изгиба отличаются между собой (рис. 3).

В таблице представлены результаты проведенных числовых экспериментальных исследований согласно закону распределения прогиба образцов бумаги с различными значениями приведенной величины коэффициента жесткости $\xi^{0}=10^{2}, \xi=1,0 ; 1,1 ; 1,2 \mathrm{Hм}$ по длине участка провисания $\left(0 \leq x \leq l_{\text {Пчо }}\right)$.

Зависимость закона распределения прогиба образцов бумаги с различными значениями коэффициента жесткости

\begin{tabular}{|c|c|c|c|c|c|c|c|}
\hline $100 x, \mathrm{M}$ & 0 & 0,5 & 1 & 1,5 & 2 & 2,5 & 3,0 \\
\hline & \multicolumn{7}{|c|}{$\xi^{0}=1,0$} \\
\cline { 2 - 8 } & 0 & 0,50 & 1,79 & 3,59 & 5,67 & 7,88 & 10,13 \\
\cline { 2 - 7 } & \multicolumn{7}{|c|}{$\xi^{0}=1,1$} \\
\hline & $000 z(x), \mathrm{M}$ & 0,55 & 1,97 & 3,94 & 6,23 & 8,66 & 11,13 \\
\cline { 2 - 8 } & \multicolumn{7}{|c|}{$\xi^{0}=1,2$} \\
\cline { 2 - 8 } & 0 & 0,60 & 2,15 & 4,30 & 6,80 & 9,45 & 12,15 \\
\hline
\end{tabular}

Из таблицы видно, что увеличение значения параметра $\xi^{0}$ приводит к возрастанию величины прогиба. В свою очередь увеличение значения параметра $\xi^{0}$ может произойти за счет увеличения значения параметров $\gamma$ и $b h$ или уменьшения значения коэффициента жесткости $E J_{Y}$ материала.

В результате, обобщая полученные данные исследований, предлагается экспериментально- теоретическая методика установления коэффициента жесткости на изгиб бумаги. Если известны экспериментальные значения прогиба $z\left(x_{0}\right)$, соответствующие некоторой точке $x_{0}$ заданного образца бумаги, то, используя эти данные значения, с помощью формулы (6) можно найти приведенное значение коэффициента жесткости материала:

$$
\xi=\frac{z\left(x_{0}\right)}{\frac{\left(l_{\text {ПчО }} x_{0}\right)^{2}}{4}+l_{\text {ПчО }} \frac{x_{0}^{2}}{6}-\frac{x_{0}^{4}}{24}} .
$$

Отсюда найдем значение коэффициента жесткости материала:

$$
E J_{Y}=\frac{1}{z\left(x_{0}\right)}\left[\frac{\left(l_{\Pi ч \mathrm{O}} x_{0}\right)^{2}}{4}+l_{\text {ПЧ }} \frac{x_{0}^{2}}{6}-\frac{x_{0}^{4}}{24}\right] \text {. }
$$

Например, если в поперечном сечение $x_{0}=0,015$ м прогиб принимает значение $z\left(x_{0}\right)=$ $=-0,0358 \mathrm{M}$, то приведенное значение жесткости материала равняется $\xi^{0}=1,0$.

Если в том же поперечном сечении $x_{0}$ прогиб принимает значение $z\left(x_{0}\right)=-0,039$ м, то приведенное значение жесткости материала $\xi^{0}=1,1$.

Заключение. Разработанные методы теоретического и экспериментального исследования внутренних сил, деформации изгиба и коэффициента жесткости на изгиб могут быть использованы при прогнозировании свойств новых видов бумаги, что даст возможность разработать оптимальную технологию их производства с заданным уровнем деформационных и прочностных свойств, а значит, максимально удовлетворить требования полиграфических предприятий.

\section{Список литературы}

1. Кулешов А. В., Смолин А. С. Бумагообразующие свойства вторичных волокнистых материалов // Химия растительного сырья. 2008. № 2. С. 109-112.

2. Галимова 3. К., Бабаханова Х. А. Влияние характера помола на прочность бумаги // Technical science and innovation. 2019. № 2. C. 100-105.

3. Гормаков А. Н., Иванова В. С. Деформация конструктивных материалов: учеб.-метод. пособие. Томск: Изд-во ТПУ, 2004. 15 с.

4. Шахкельдян Б. Н., Загаринская Л. А. Полиграфические материалы. М.: Книга, 1988. 330 с.

5. Мирзаева М. Б., Бабаханова Х. А. Влияние процесса размола бумажной массы на механическую прочность бумаг // Проблемы текстиля. 2012. С. 163-168.

6. Комаров В. И., Казаков Я. В. Анализ механического поведения целлюлозно-бумажных материалов при приложении растягивающей нагрузки // Лесной вестник. 2000. № 3. С. 52-62.

7. Брайловская Е. Д., Сосновская А. П., Эмдин А. Г. Оценка качества печатной бумаги. М.: Книга, 1979. $116 \mathrm{c}$.

8. Комаров В. И. «Критическая» длина волокна - фактор, определяющий деформативность и прочность целлюлозно-бумажных материалов // Лесной журнал. 1993. № 4. С. 79-83.

9. Айзерман М. А. Классическая механика. М.: Наука, 1980. 367 с.

10. Пановко Я. Г. Механика деформируемого твердого тела. М.: Наука, 1985. 288 с.

11. Виттенбург Й. Динамика систем твердых тел. М.: Мир, 1980. 292 с.

12. Галин Л. А. Контактные задачи теории упругости и вязкоупругости. М.: Наука, 1980. 303 с.

13. Комаров В. И. Жесткость при изгибе целлюлозно-бумажных материалов. Анализ методов измерения и влияния технологических факторов // Лесной журнал. 1994. № 3. С. 112-142. 
14. Работнов Ю. Н. Механика деформируемого тела. М.: Наука, 1988. 713 с.

15. Yuldashev J. Q., Rayimberdieva D. X., Mirxojayev M. M., Atambaev D. D. Analysis of Modern Sportswear Materials // International Journal of Advanced Research in Science, Engineering and Technology. INDIA. Vol. 6, issue 3, March 2019. P. 8535-8540.

16. Абдуллаев У. Т., Мирхаджаев М. М., Эргашов М. Метод оценки сопротивление на изгиб текстильных нитей // Научно-технический журнал Наманганского инженерно-технологического института. 2020. № 1. С. 6-10.

\section{References}

1. Kuleshov A. V., Smolin A. S. Paper-forming properties of secondary fibrous materials. Khimiya rastitel'nogo syr'ya [Chemistry of vegetable raw materials], 2008, no. 2, pp. 109-112 (In Russian).

2. Galimova Z. K., Babakhanova Kh. A. Influence of the nature of grinding on paper strength. Technical science and innovation, 2019, no. 2, pp. 100-105 (In Russian).

3. Gormakov A. N., Ivanova V. S. Deformatsiya konstruktivnykh materialov [Deformation of structural materials]. Tomsk, TPU Publ., 2004. 15 p.

4. Shakhkel'dyan B. N., Zagarinskaya L. A. Poligraficheskiye materialy [Polygraphic materials]. Moscow, Kniga Publ., 1988. 330 p.

5. Mirzaeva M. B., Babakhanova Kh. A. Influence of the pulp grinding process on the mechanical strength of papers. Problemy tekstilya [Textile problems], 2012, pp. 163-168 (In Russian).

6. Komarov V. I., Kazakov Ya. V. Analysis of the mechanical behavior of pulp and paper materials under tensile load. Lesnoy vestnik [Forest bulletin], 2000, no. 3, pp. 52-62 (In Russian).

7. Braylovskaya E. D., Sosnovskaya A. P., Emdin A. G. Otsenka kachestva pechatnoy bumagi [Assessing the quality of printing paper]. Moscow, Kniga Publ., 1979. 116 p.

8. Komarov V. I. "Critical" fiber length - a factor that determines the deformability and strength of pulp and paper materials. Lesnoy zhurnal [Forest Journal], 1993, no. 4, pp. $79-83$ (In Russian).

9. Ayzerman M. A. Klassicheskaya mekhanika [Classic mechanics]. Moscow, Nauka Publ., 1980. 367 p.

10. Panovko Ya. G. Mekhanika deformiruyemogo tverdogo tela [Deformable solid mechanics]. Moscow, Nauka Publ., 1985. 288 p.

11. Vittenburg J. Dinamika sistem tverdykh tel [Dynamics of systems of rigid bodies]. Moscow, Mir Publ., 1980. 292 p.

12. Galin L. A. Kontaktnyye zadachi teorii uprugosti $i$ vyazkouprugosti [Contact problems of the theory of elasticity and viscoelasticity]. Moscow, Nauka Publ., 1980. 303 p.

13. Komarov V. I. Flexural stiffness of pulp and paper materials. Analysis of measurement methods and the influence of technological factors. Lesnoy zhurnal [Forest Journal], 1994, no. 3, pp. 112-142 (In Russian).

14. Rabotnov Yu. N. Mekhanika deformiruyemogo tela [Mechanics of a deformable body]. Moscow, Nauka Publ., 1988. 713 p.

15. Yuldashev J. Q., Rayimberdieva D. X., Mirxojayev M. M., Atambaev D. D. Analysis of Modern Sportswear Materials. International Journal of Advanced Research in Science, Engineering and Technology. INDIA, vol. 6, issue 3, March 2019, pp. 8535-8540 (In English).

16. Abdullaev U. T., Mirkhadzhaev M. M., Ergashov M. Method for assessing the flexural resistance of textile threads. Nauchno-tekhnicheskiy zhurnal Namanganskogo inzhenerno-tekhnologicheskogo instituta [Scientific and technical journal of the Namangan Engineering and Technological Institute], 2020, no. 1, pp. 6-10 (In Russian).

\section{Информация об авторах}

Эргашов Махаматрасул - доктор технических наук, профессор кафедры «Машиностроение и сервис». Ташкентский институт текстильной и легкой промышленности $(100100$, г. Ташкент, ул. Шохжахон, 5, Республика Узбекистан). E-mail: maxamatrasul@bk.ru

Бабаханова Халима Абишевна - доктор технических наук, профессор кафедры «Технология полиграфического и упаковочного процессов». Ташкентский институт текстильной и легкой промышленности (100100, г. Ташкент, ул. Шохжахон, 5, Республика Узбекистан). E-mail: halima300@inbox.ru

Абдуллаев Улугбек Туланбаевич - кандидат технических наук, доцент кафедры «Машиностроение и сервис». Ташкентский институт текстильной и легкой промышленности $(100100$, г. Ташкент, ул. Шохжахон, 5, Республика Узбекистан). E-mail: abdullaev1975@mail.ru 
Садриддинов Акмал Абдулло угли - докторант кафедры «Технология полиграфического и упаковочного процессов». Ташкентский институт текстильной и легкой промышленности (100100, г. Ташкент, ул. Шохжахон, 5, Республика Узбекистан). E-mail: sadriddinov@mail.ru

Громыко Ирина Григорьевна - кандидат технических наук, доцент, заведующая кафедрой полиграфических производств. Белорусский государственный технологический университет (220006, г. Минск, ул. Свердлова, 13a, Республика Беларусь). E-mail: gromyko@belstu.by

\section{Information about the authors}

Ergashov Maxamatrasul - DSc (Engineering), Professor, the Department of Mechanical Engineering and Service. Tashkent Institute of Textile and Light Industry (5, Shokhzhakhon str., 100100, Tashkent, Republic of Usbekistan).E-mail: maxamatrasul@bk.ru

Babakhanova Khalima Abishevna - DSc (Engineering), Professor, the Department of Technology of Printing and Packing Processes. Tashkent Institute of Textile and Light Industry (5, Shokhzhakhon str., 100100, Tashkent, Republic of Usbekistan). E-mail: halima300@inbox.ru

Abdullayev Ulugbek Tulanbayevich - PhD (Engineering), Assictant Professor, the Department of Mechanical Engineering and Service. Tashkent Institute of Textile and Light Industry (5, Shokhzhakhon str., 100100, Tashkent, Republic of Usbekistan).E-mail: abdullaev1975@mail.ru

Sadriddinov Akmal Abdullo ugli - post-doktoral student, the Department of Technology of Printing and Packing Processes. Tashkent Institute of Textile and Light Industry (5, Shokhzhakhon str., 100100, Tashkent, Republic of Usbekistan). E-mail: sadriddinov@mail.ru

Gromyko Irina Grigor'yevna - PhD (Engineering), Associate Professor, Head of the Department of Printing Production. Belarusian State Technological University (13a, Sverdlova str., 220006, Minsk, Republic of Belarus). E-mail: gromyko@belstu.by 